\title{
CYTOGENETIC AND MOLECULAR RELATIONSHIPS BETWEEN ZARUDNY'S ROCK SHREW (CROCIDURA ZARUDNYI; MAMMALIA: SORICOMORPHA) AND EURASIAN TAXA
}

\author{
Sylvain Dubey,* Petra Nová, Peter Vogel, and Vladimír Vohralík \\ Department of Ecology and Evolution, University of Lausanne, 1015 Lausanne, Switzerland (SD, PV) \\ Department of Zoology, Charles University, Vinicná 7, CZ-128 44 Prague 2, Czech Republic (PN, VV)
}

\begin{abstract}
We karyotyped and sequenced 1,140 base pairs of the mitochondrial DNA cytochrome $b$ of a specimen of Zarudny's rock shrew (Crocidura zarudnyi) from Baluchestan, southeastern Iran, to clarify its cytogenetic and molecular relationships with other Eurasian species of Crocidura. According to the karyotype $(2 \mathrm{~N}=40, \mathrm{FN}=$ 50), Zarudny's rock shrew belongs to the group of the lesser white-toothed shrew (C. suaveolens), which is different from other known crocidurine karyotypes, considering the combination of the diploid and fundamental number of chromosomes. Molecular results revealed that $C$. zarudnyi is included in a monophyletic clade with the $C$. suaveolens group, where it is a sister taxon to the others (mean Kimura 2-parameter distance $=9.7 \%$ ).
\end{abstract}

Key words: Crocidura, cytogenetics, Iran, mitochondrial DNA, systematics

Within the family Soricidae (order Soricomorpha), Crocidura is the largest genus, represented by about 170 species distributed throughout Eurasia and Africa. Not surprisingly, the taxonomic status of many rare species commonly remains uncertain (Hutterer 2005). This is also the case for some shrews in the Middle East, for example, Zarudny's rock shrew (Crocidura zarudnyi Ognev, 1928), whose original description was based on a single specimen collected from the modern-day Iranian province of Baluchestan, on the Pakistan border. Ellerman and Morrison-Scott (1951) and Lay (1967) considered Zarudny's rock shrew to be a subspecies of the pale gray shrew (C. pergrisea Miller, 1913). However, later authors who studied additional specimens of Middle Eastern white-toothed shrews that also included specimens of C. zarudnyi from Iran, Afganistan, and Pakistan considered C. zarudnyi to be a valid species (e.g., Hassinger 1970, 1973; Jenkins 1976; Kryštufek and Vohralík 2001; Spitzenberger 1971). This view also was adopted by Hutterer (1993, 2005) and Wolsan and Hutterer (1998). The known distribution of $C$. zarudnyi includes southeastern Iran, southwestern Pakistan, and Afghanistan (Hutterer 2005). So far, fewer than 20 specimens of the species have been

* Correspondent: sylvain.dubey@unil.ch

(C) 2007 American Society of Mammalogists www.mammalogy.org collected. During a field trip to Iran, PN and VV collected a very small shrew identified as $C$. zarudnyi; in this paper we describe its karyotype and phylogenetic position within Eurasian crocidurine shrews.

\section{Materials ANd Methods}

On 12 April 2000, we collected a specimen of Crocidura in Pir Sohrab, Baluchestan, southeastern Iran $\left(25^{\circ} 45^{\prime} \mathrm{N}, 60^{\circ} 50^{\prime} \mathrm{E}\right.$, elevation $130 \mathrm{~m}$ ). The shrew was trapped in an abandoned 2-m-deep lair dug by the crocodile Crocodylus palustris in the sheer bank of a temporarily dry riverbed. The habitat was a sandy-loess riverbank covered by dense reeds. We used the direct treatment of bone marrow cells for karyotype analysis, following the standard method modified after Ford and Hamerton (1956). The slides were conventionally stained by $5 \%$ Giemsa, and 50 metaphase chromosome preparations were analyzed. We used the chromosome nomenclature of Zima and Král (1984). The voucher specimen and microscopic slides are deposited in the Department of Zoology, Charles University, Prague, Czech Republic, under no. I-89.

DNA was extracted from muscle tissue using a Qiagen kit (QIA Amp DNA Mini Kit; Qiagen, Valencia, California). Double-stranded DNA amplifications of the mitochondrial cytochrome- $b(C y t b)$ gene were performed with the primers Cytb3 (5'-TAT TCT CCC CAG ACA TAT TAG G-3'), Cytb6 (5'-CTT GAA ACA TGA AAC ATT GG-3'), and Cytb7 (5'-AAT AGA AAA TAT CAT TCT GG-3'), which are specific to the genus Crocidura and were designed in our laboratory, and 
L14724, H15149, and H15915 (Irwin et al. 1991). Amplification conditions consisted of 35 thermal cycles with the primers L14724/H15149, Cytb6/Cytb7, and Cytb3/H15915 under the following conditions: $30 \mathrm{~s}$ denaturation at $94^{\circ} \mathrm{C}, 45 \mathrm{~s}$ annealing at $50^{\circ} \mathrm{C}$, and $60 \mathrm{~s}$ extension at $72^{\circ} \mathrm{C}$. Polymerase chain reaction products were then electrophoresed on a $1 \%$ agarose gel, visualized with ethidium bromide staining to verify polymerase chain reaction quality, and purified by centrifugal dialysis using the QIAquick PCR Purification Kit (Qiagen) according to the manufacturer's instructions. Cycle sequencing was performed on a $10-\mu 1$ sample of $1-3 \mu \mathrm{l}$ of amplified DNA, $1 \mu \mathrm{l}$ of $10 \mu \mathrm{M}$ primer, and $4 \mu \mathrm{l}$ of ABI PRISM Dye Terminator 1 (Perkin Elmer, Boston, Massachusetts); and water was added to bring the reaction volume to $10 \mu \mathrm{l}$. Reaction sequences were visualized on an ABI 3100 genetic analyzer (Applied Biosystems, Foster City, California).

To clarify the genetic relationship between $C$. zarudnyi (AY925211) and other Eurasian crocidurine shrews, additional DNA sequences were obtained from GenBank (Appendix I). Nucleotide sequences were aligned by eye; no insertions or deletions were observed.

Two methods of phylogenetic analyses were carried out using PAUP version 4.0b10 PPC (Swofford 1998). Maximumparsimony analyses were performed with the following options: heuristic search, stepwise addition of sequences, 10 replicates of random addition of taxa, and tree-bisection-reconnection branch swapping (Swofford 1998). Support values were estimated using 1,000 bootstrap resamples using the same heuristic settings. All codon positions were equally weighted. The tree was rooted using sequences of the Eurasian water shrew (Neomys fodiens; DQ065611) and the Caucasian pygmy shrew (Sorex volnuchini; DQ065610).

For maximum-likelihood analysis, we 1st used likelihood ratio tests implemented via the computer program MODELTEST 3.06 to choose the mutation model that best fits the data according to the protocol of Posada and Crandall (1998). Tests were conducted on the total fragment of 1,140 base pairs (bp). The $\mathrm{TRn}+\mathrm{I}+\mathrm{G}$ model was selected. Maximum-likelihood analyses were then performed, assuming this model. Estimated parameters from the data were as follows: the proportion of invariable sites is 0.5435 , the unequal distribution of rates at variable sites $(\gamma)$ is 1.5224 , and 4 different substitution types $($ rate $[\mathrm{A}-\mathrm{C}]=0.9483$, rate $[\mathrm{A}-\mathrm{G}]=9.9527$, rate $[\mathrm{A}-\mathrm{T}]=$ 1.2830 , rate $[\mathrm{C}-\mathrm{G}]=0.3757$, rate $[\mathrm{G}-\mathrm{T}]=1.0000$, rate $[\mathrm{C}-\mathrm{T}]=20.6991)$. Maximum-likelihood bootstrap analyses (1,000 replicates) were performed using the software phyML version 2.4.4. (Guindon and Gascuel 2003).

Bayesian analysis was conducted using MrBayes version 3.0 b4 (Huelsenbeck and Ronquist 2001), which performs Metropolis-coupled Markov chain Monte Carlo analysis. A GTR model was used with an among-site rate variation following a gamma distribution. The Markov chain was run for 1,000,000 generations and sampled once every 100 generations; burn-in was set at 100,000 generations. To assure convergence in the Bayesian analyses, 2 independent runs were performed and compared.

The molecular clock hypothesis was tested following Posada and Crandall (1998). Estimation of divergence time from the molecular data was performed according to the calibration developed for the Soricidae by Fumagalli et al. (1999).

\section{Results}

Morphological identification.-Our specimen was a tiny, long-tailed, and gracile shrew. Its mass was $5 \mathrm{~g}$, head and body length was $68 \mathrm{~mm}$, tail length (taken from the anus, without terminal hairs) was $47 \mathrm{~mm}$, hind-foot length (without claws) was $12.0 \mathrm{~mm}$, and ear length was $9 \mathrm{~mm}$. The ratio of tail length to head and body length was therefore $69 \%$. The specimen was an adult female with signs of previous lactation but only slightly worn teeth. Principal skull measurements were as follows: condylobasal length $=16.9 \mathrm{~mm}$, zygomatic breadth $=$ $5.5 \mathrm{~mm}$, maximum braincase breadth $=7.7 \mathrm{~mm}, \mathrm{P} 4-\mathrm{M} 3$ length $=$ $4.5 \mathrm{~mm}$, height of the mandible taken across the coronoid process $=4.4 \mathrm{~mm}$. The back was pale brown with reddish shades and the belly was whitish; there was no clear demarcation line. The tail was bicolored, with the upper part being pale brown and the under part whitish gray. White hairs covered fairly conspicuous ears.

Species identification was based mostly on comparison with the very detailed description of the type specimen by Ognev (1928). The coloration, body and skull measurements, and shape of the skull of our specimen fit the type description well. Measurements of our specimen were also consistent with those given by Hassinger (1970) for the series of C. zarudnyi from Iran, West Pakistan, and Afganistan (Table 1).

Other species reported from the Middle East, such as the lesser white-toothed shrew (C. suaveolens (Pallas, 1811)), the pale gray shrew (C. pergrisea Miller, 1913), the lesser rock shrew (C. serezkyensis Laptev, 1929), and the Iranian shrew (C. susiana Redding and Lay, 1978), were excluded for the following reasons. $C$. pergrisea (type locality in Kashmir) is larger, with its condylobasal length being at least $19.0 \mathrm{~mm}$ (Hassinger 1973; Jenkins 1976; see Table 1). C. susiana (type locality in Khuzestan, northwestern Iran) is rather large, its condylobasal length exceeds $20.5 \mathrm{~mm}$, and the tail is not bicolored (Redding and Lay 1978). In Table 1 it is clearly demonstrated that there is no overlap between available specimens of $C$. zarudnyi and the type series of $C$. pergrisea and $C$. susiana in any measurement. $C$. serezkyensis (type locality in the Pamir Mountains, Tajikistan) has a deeply grooved posterior edge on the 3rd upper unicuspid (Zaitsev 1993). The 3rd upper unicuspid of our specimen was grooved only very slightly as is common, for example, in $C$. suaveolens. Further, C. suaveolens (type locality Crimea, Ukraine) can be excluded because the divergence in $\mathrm{Cytb}$ sequence observed between our specimen of $C$. zarudnyi and $C$. suaveolens from Crimea (Kimura 2-parameter distance $=9.75 \%$ ) clearly points out that they belong to different species.

Karyological result.-In the examined specimen, the karyotype diploid number $(2 \mathrm{~N})=40$ chromosomes, fundamental number $(\mathrm{FN})=50$ was found. Fifteen autosomal pairs were gradually smaller acrocentrics and 4 pairs of autosomes were biarmed (Fig. 1). One pair of biarmed autosomes was subtelocentric, and was the largest of the entire group. The 
Table 1.-Measurements (mm) of Crocidura zarudnyi, C. pergrisea, and C. susiana from the Middle East. Means are given with minimum and maximum values in parentheses.

\begin{tabular}{|c|c|c|c|c|c|}
\hline & \multicolumn{3}{|c|}{ C. zarudnyi } & \multirow[b]{2}{*}{ C. pergrisea, type series, $n=3^{\mathrm{d}}$} & \multirow[b]{2}{*}{ C. susiana, type series, $n=4-7^{\mathrm{e}}$} \\
\hline & Holotype $^{a}$ & Our specimen $^{\mathrm{b}}$ & Series, $n=11-15^{\mathrm{c}}$ & & \\
\hline Total length & 108.3 & 115 & $104.7(92-116)$ & $127.3(125-129)$ & $138.3(134-148)$ \\
\hline Condyloincisive length & 18.1 & 17.5 & $18.1(16.9-19.0)$ & - & $20.7(20.6-21.0)$ \\
\hline Condylobasal length & - & 16.9 & $-(16.5-18.2)$ & $19.1(19.0-19.2)$ & - \\
\hline Maximum braincase breadth & 8.3 & 7.7 & $7.9(7.5-8.3)$ & $8.8(8.8-8.8)$ & $9.3(9.0-9.4)$ \\
\hline
\end{tabular}

a Ognev 1928

b This study.

${ }^{\mathrm{c}}$ Hassinger 1970 (specimens from Iran, West Pakistan, and Afganistan).

d Spitzenberger 1971.

${ }^{\mathrm{e}}$ Redding and Lay 1978.

other 3 pairs were medium sized, with various positions of the centromere: 1 pair was subtelocentric, 1 submetacentric, and 1 metacentric. The $\mathrm{X}$ chromosome was a large submetacentric.

Phylogenetic analyses.-The 37 sequences of 1,140 bp used in this study showed 490 variable sites of which 417 were parsimony-informative; we found 1 most-parsimonious tree with a length of 2,339 steps. Interspecific pairwise sequence divergences between Crocidurinae species (Kimura 2-parameter distances) varied from 0.032 (savanna path shrew [C. viaria] versus African giant shrew [C. olivieri]) to 0.205 (Canarian shrew [C. canariensis] versus Dsinezumi shrew [C. Asinezumi]). Pairwise sequence divergences between $C$. zarudnyi and other species of Crocidura varied from 0.089 ( $C$. suaveolens 8 ) to 0.182 (C. olivieri).

The phylogenetic relationship among haplotypes is given in Fig. 2. In all phylogenetic analyses, 6 well-defined clades were found. The 1 st included the samples of the $C$. suaveolens group (Asian lesser white-toothed shrew [C. shantungensis] and C. suaveolens; for more details about the systematics of this group, see Dubey et al. [2006]) and C. zarudnyi, $2 \mathrm{~N}=40, \mathrm{FN}=$ 50 , which is the sister taxon to the latter species; this was

\section{His}
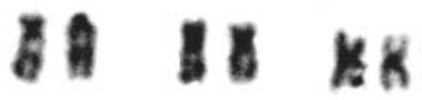

OB
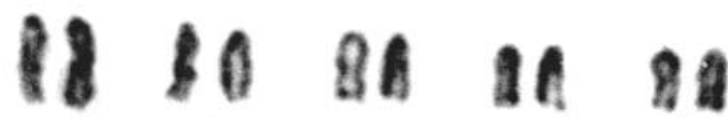

an

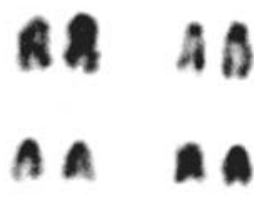

10
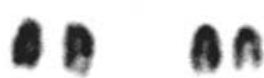

16

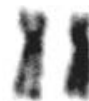

FIG. 1.-Conventionally stained karyotype of Crocidura zarudnyi, collected 12 April 2000 in Baluchestan, Iran. The 2 large chromosomes at bottom right are the $\mathrm{X}$ chromosomes. supported by maximum-likelihood and maximum-parsimony bootstrap values of $100 \%$, and Bayesian posterior probability of 1.0. The 2 nd included samples with $2 \mathrm{~N}=40, \mathrm{FN}=56$ chromosomes $(C$. dsinezumi, the Ussuri white-toothed shrew [C. lasiura], and Kuroda's shrew [C. kurodai]; bootstrap of $100 \%$ and $94 \%$, respectively for maximum likelihood and maximum parsimony, and Bayesian posterior probability of 1.0). The 3rd included samples of Horsfield's shrew (C. horsfieldii; $2 \mathrm{~N}=40$, $\mathrm{FN}=54)$, and the lesser Ryukyu shrew (C. watasei; $2 \mathrm{~N}=26$, $\mathrm{FN}=52$ ), and was supported by bootstrap values for maximum likelihood and maximum parsimony of $100 \%$, and Bayesian posterior probability of 1.0 . The 4 th included the samples with $2 \mathrm{~N}=38, \mathrm{FN}=56$ chromosomes (the black-footed shrew $[C$. nigripes] and the thick-tailed shrew [C. brunnea]; bootstrap values of $100 \%$ and $89 \%$ for maximum likelihood and maximum parsimony, and Bayesian posterior probability of 1.0$)$. The 5th included the samples with $2 \mathrm{~N}=36, \mathrm{FN}=56$ chromosomes $(C$. canariensis and the Sicilian shrew [C. sicula]; bootstrap values of $90 \%$ and $63 \%$ for maximum likelihood and maximum parsimony, and Bayesian posterior probablity of 0.8 ). The last clade included the samples of $C$. olivieri and $C$. viaria $(2 \mathrm{~N}=50$, $\mathrm{FN}=66$; maximum-likelihood and maximum-parsimony bootstrap values of $100 \%$, and Bayesian posterior probability of 1.0). Nevertheless, the phylogenetic relationships between these 6 clades and the other samples $(C$. theresae, $C$. russula, $C$. zimmermanni, $C$. fuliginosa, and $C$. orii) remain uncertain.

A likelihood ratio test led to the acceptance of the molecular clock hypothesis for the whole sample $\left(\chi^{2}=44.52\right.$, d.f. $=35$, $P>0.05)$. Ln-likelihood values are 10,390.52 and 10,368.26, respectively, for the best trees, with and without the molecular clock assumption. On the basis of the calibration of Fumagalli et al. (1999), we estimate the divergence time between C. zarudnyi and the $C$. suaveolens group to be 2.68 million years, with the $95 \%$ confidence interval ranging from 2.1 to 3.38 million years.

\section{Discussion}

Our specimen of $C$. zarudnyi had a karyotype that appears to be identical to that of the $C$. suaveolens group based on the combination of the diploid and fundamental number of 


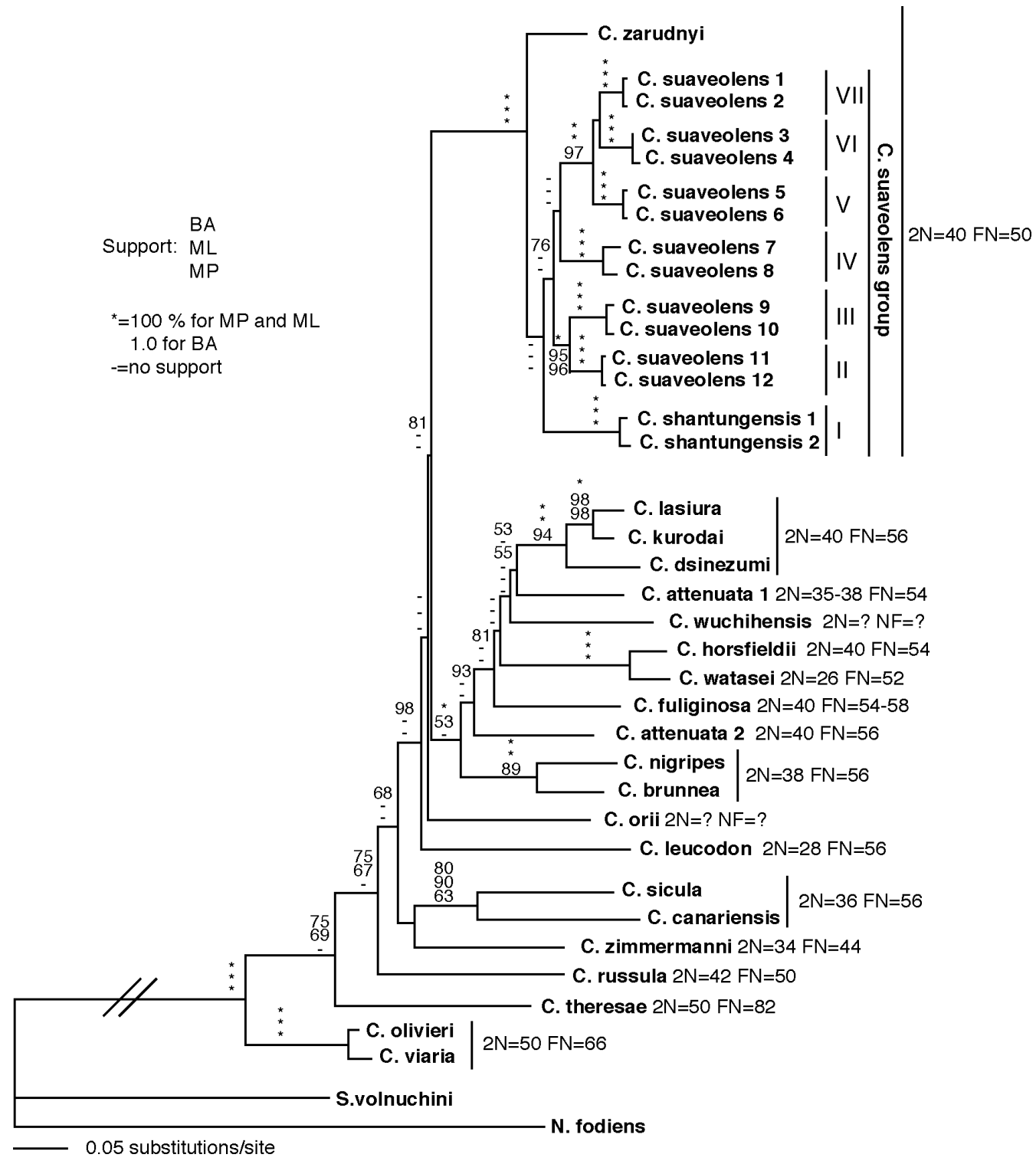

Fig. 2.-Phylogeny of the 1,140-bp Cytb fragment analyzed with maximum likelihood, using the GTR $+\mathrm{I}+\mathrm{G}$ model of substitution and treebisection-reconnection branch swapping. Values in branches are bootstrap indices of support for maximum-parsimony (MP) and maximumlikelihood (ML) analyses (percentage of 1,000 replications for each of the 10 random orders of stepwise addition of sequences), and Bayesian posterior probabilities (BA). Clade numbers of Crocidura suaveolens group are those described in Dubey et al. (2006). Codes are as in Appendix I.

chromosomes $(2 \mathrm{~N}=40, \mathrm{FN}=50)$. In fact, this karyotype is known not only from C. suaveolens (Catzeflis et al. 1985; Grafodatsky et al. 1988; Meylan 1966; Meylan and Hausser 1974; Reumer and Meylan 1986), but also from related forms such as Gueldenstaedt's shrew (C. gueldenstaedtii-Catzeflis et al. 1985; Grafodatsky et al. 1988) and C. sibirica (Grafodatsky et al. 1988) that may both be assigned to $C$. suaveolens (Dubey et al. 2006). Moreover, C. shantungensis has the same karyotype (Iwasa et al. 2001). In contrast, all other crocidurine shrews exhibit different combinations of diploid and fundamental numbers of chromosomes, including $C$. serezkyensis (assigned to C. pergrisea) reported from southwestern Azerbaijan by Grafodatsky et al. (1988), which has a karyotype of $2 \mathrm{~N}=22$ and $\mathrm{FN}=34$. Vogel et al. (2003) concluded that a shared karyotype in the genus Crocidura is a synapomorphy that signals a monophyletic relationship. If this is correct, then $C$. zarudnyi should theoretically cluster together with members of the $C$. suaveolens group in the phylogenetic analyses.

Our molecular results confirm this phylogenetic relationship. In fact, $C$. zarudnyi is included in a highly supported monophyletic clade (maximum-parsimony and maximum-likelihood bootstrap values of $100 \%$, and Bayesian posterior probablity of 1.0), the $C$. suaveolens group, where it is placed as sister taxon to the other members of this clade (mean Kimura 2-parameter distance between $C$. zarudnyi and $C$. suaveolens group $=0.097$ ). The separation between $C$. zarudnyi and its close relatives goes back to the Middle Pliocene (2.68 million years ago). 
The other taxa analyzed, such as the Asian shrews, which show a similar diploid number of chromosomes but a different fundamental number, are clearly separated from this group (mean Kimura 2-parameter distance between $C$. suaveolens group and other $2 \mathrm{~N}=40=0.163$ )

In conclusion, the particular phylogenetic position of $C$. zarudnyi makes this a key species in understanding the evolution of the $C$. suaveolens group. However, its relationships with the other little-known Middle Eastern white-toothed shrews, $C$. pergrisea and $C$. susiana, remain obscure in the absence of further cytogenetic and molecular studies.

\section{ACKNOWLEDGMENTS}

The paper was partly sponsored by Grantová Agentura České Republiky (GACR) (grant 206/05/2334) and Ministerstvo Školstvi Mládeže a Tělovýchovy České Republiky (MSMT CR) (project 0021620828). We acknowledge N. Di Marco for laboratory facilities.

\section{Literature Cited}

Catzeflis, F., T. Maddalena, S. Hellwing, and P. Vogel. 1985. Unexpected findings on the taxonomic status of east Mediterranean Crocidura-russula auct. (Mammalia, Insectivora). Zeitschrift für Säugertierkunde 50:185-201.

Dubey, S., M. Zaitsev, J.-F. Cosson, A. Abdukadier, and P. Vogel. 2006. Pliocene and Pleistocene diversification and multiple refugia in a Eurasian shrew (Crocidura suaveolens group). Molecular Phylogenetic and Evolution 38:635-647.

Ellerman, J. R., AND T. C. S. Morrison-Scott. 1951. Checklist of Palaearctic and Indian mammals 1758 to 1946. British Museum (Natural History), London, United Kingdom.

Fontanillas, P., A. Depraz, M. S. Giorgi, and N. Perrin. 2005. Nonshivering thermogenesis capacity associated to mitochondrial DNA haplotypes and gender in the greater white-toothed shrew, Crocidura russula. Molecular Ecology 14:661-670.

Ford, C. E., AND J. L. HAMERTON. 1956. A colchicine, hypotonic citrate, squash sequence for mammalian chromosomes. Stain Technology 31:247-251.

Fumagalli, L., P. Taberlet, D. T. Stewart, L. Gielli, J. Hausser, AND P. Vogel. 1999. Molecular phylogeny and evolution of Sorex shrews (Soricidae: Insectivora) inferred from mitochondrial DNA sequence data. Molecular Phylogenetics and Evolution 11: 222-235.

Grafodatsky, A. S., S. I. Radzhabli, A. V. Sharshov, and M. V. ZAITSEV. 1988. Karyotypes of five Crocidura species of the USSR fauna. Tsitologiya 30:1247-1250.

Guindon, S., AND O. Gascuel. 2003. A simple, fast, and accurate algorithm to estimate large phylogenies by maximum likelihood. Systematic Biology 52:696-704.

HASSINGER, J. D. 1970. Shrews of the Crocidura zarudnyi-pergrisea group with descriptions of a new subspecies. Fieldiana: Zoology 58:5-8.

HASSINGER, J. D. 1973. A survey of the mammals of Afganistan resulting from the 1965 Street expedition (excluding bats). Fieldiana: Zoology 60:1-195.

Huelsenbeck, J. P., And F. Ronquist. 2001. MrBayes: Bayesian inference of phylogenetics trees. Bioinformatics Application Notes 17:754-755.
Hutterer, R. 1993. Order Insectivora. Pp. 69-130 in Mammal species of the world: a taxonomic and geographic reference (D. E. Wilson and D. M. Reeder, eds.). 2nd ed. Smithsonian Institution Press, Washington, D.C.

Hutterer, R. 2005. Order Soricomorpha. Pp. 220-311 in Mammal species of the world: a taxonomic and geographic reference (D. E. Wilson and D. M. Reeder, eds.). 3rd ed. Johns Hopkins University Press, Baltimore, Maryland.

Irwin, D. M., T. D. Kocher, AND A. C. WiLson. 1991. Evolution of the cytochrome-b gene of mammals. Journal of Molecular Evolution 32:128-144.

Iwasa, M. A., S. Ohdachi, S. H. Han, H. S. Оh, H. Abe, AND H. SUZUKI. 2001. Karyotype and RFLP of the nuclear rDNA of the Crocidura sp. on Cheju Island, South Korea. Mammalia 65: 451-459.

JENkINS, P. D. 1976. Variation in Eurasian shrews of the genus Crocidura (Insectivora: Soricidae). Bulletin of the British Museum (Natural History), Zoology 30:269-309.

KryštufeK, B., And V. Vohralík. 2001. Mammals of Turkey and Cyprus. Introduction, checklist, Insectivora. Zgodovinsko društvo za južno Primorsko, Koper, Slovenia.

LAY, D. M. 1967. A study of the mammals of Iran resulting from Street expedition of 1962-63. Fieldiana: Zoology 54:1-282.

Meylan, A. 1966. Données nouvelles sur les chromosomes des Insectivores européens (Mamm.). Revue Suisse de Zoologie 73: 548-558.

Meylan, A., AND J. Hausser. 1974. Position cytotaxonomique de quelques musaraignes du genre Crocidura au Tessin (Mammalia, Insectivora). Revue Suisse de Zoologie 81:701-710.

Ognev, S. I. 1928. Zveri vostochnoi Evropy i severnoi Azii. Tom I. Nasekomoyadnye i letuchie myshi. (The mammals of the eastern Europe and the northern Asia. Vol. I. Insectivora and Chiroptera.) Glavnauka, Moscow, Russia (in Russian).

Ohdachi, S. D., M. Hasegawa, M. A. Iwasa, P. Vogel, T. Oshida, L. K. LIN, AND H. ABE. 2006. Molecular phylogenetics of soricid shrews (Mammalia) based on mitochondrial cytochrome $b$ gene sequences: with special reference to the Soricinae. Journal of Zoology (London) 270:777-791.

Ohdachi, S. D., M. A. Iwasa, V. A. Nesterenko, H. Abe, R. Masuda, AND W. HABERL. 2004. Molecular phylogenetics of Crocidura shrews (Insectivora) in east and central Asia. Journal of Mammalogy 85:396-403.

Posada, D., and K. A. Crandall. 1998. MODELTEST: testing the model of DNA substitution. Bioinformatics 14:817-818.

REDDING, R. W., AND D. M. LAY. 1978. Description of a new species of shrew of the genus Crocidura (Mammalia: Insectivora: Soricidae) from southwestern Iran. Zeitschrift für Säugetierkunde 43:306-310.

Reumer, J. W. F., and A. Meylan. 1986. New developments in vertebrate cytotaxonomy. 9. Chromosome numbers in the order Insectivora (Mammalia). Genetica 70:119-151.

SPITZENBERGER, F. 1971. Eine neue, tiergeographisch bemerkenswerte Crocidura (Insectivora, Mammalia) aus der Türkei. Annalen des Naturhistorischen Museums in Wien 75:539-552.

Swofford, D. L. 1998. Paup*. Phylogenetic analysis using parsimony (* and other methods). Version 4.0b1. Sinauer Associates, Inc., Publishers, Sunderland, Massachusetts.

Vogel, P., J. F. Cosson, And L. F. L. JuRAdo. 2003. Taxonomic status and origin of the shrews (Soricidae) from the Canary Islands inferred from a mtDNA comparison with the European Crocidura species. Molecular Phylogenetic and Evolution 27:271-282. 
Wolsan, M., and R. Hutterer. 1998. A list of living species of shrews. Pp. 423-448 in Evolution of shrews (J. M. Wójcik and M. Wolsan, eds.). Mammalian Research Institute, Polish Academy of Sciences, Bialowieza, Poland.

Zaitsev, M. V. 1993. Species composition and questions of systematics of whitetoothed shrews (Mammalia: Insectivora) of the fauna of USSR. Proceedings of the Zoological Institute, USSR Academy of Sciences 243:3-46 (in Russian, English summary).

ZimA, J., AND B. KRÁL. 1984. Karyotype of European mammals I. Acta scientiarum naturalium Academiae scientiarum bohemoslovacaeBrno 18(7):1-51.

Submitted 8 December 2005. Accepted 16 November 2006.

Associate Editors were Jesús E. Maldonado and Joseph F. Merritt.

\section{APPENDix I}

Species, locations of samples, accession numbers, and collection numbers for our specimens included in our phylogenetic analyses, including citations. 1. Crocidura zarudnyi, Iran, Baluchestan, AY925211, I-89, our study. 2. Crocidura suaveolens 2, Hungary, Fülophasa, DQ242541, IZEA 6732, our study. 3. Crocidura leucodon, Turkey, Altindere, DQ065609, IZEA 6040, our study. 4. Crocidura theresae, Burkina Faso, Bobo Dioulasso, DQ521043, IZEA3092, our study. 5. Crocidura olivieri, Central African Republic, Bangui, DQ521045, IZEA 2821, our study. 6. Crocidura viaria, Burkina Faso, Oursi, DQ521044, IZEA 3108, our study. 7. Sorex volnuchini, Turkey, Altindere, DQ065610, IZEA 6079, our study. 8. Neomys fodiens, Italy, Reggio, DQ065611, IZEA 5643, our study. 9. Crocidura sicula, Italy, Sicily, DQ521040, IZEA 2943, Vogel et al. 2003. 10. Crocidura canariensis, Spain, Canary Is., DQ521042, IZEA 4285, Vogel et al.
2003. 11. Crocidura zimmermanni, Greece, Crete Is., DQ521041, IZEA 2053, Vogel et al. 2003. 12. Crocidura suaveolens 3, Greece, Lesvos Is., AY843460, Dubey et al. 2006. 13. Crocidura suaveolens 4, Turkey, Vukarikisilka, AY843461, Dubey et al. 2006. 14. Crocidura suaveolens 5, Turkey, Rize, AY843498, Dubey et al. 2006. 15. Crocidura suaveolens 6, Georgia, Shulaveri, AY843500, Dubey et al. 2006. 16. Crocidura suaveolens 7, Spain, Figueras, AY843491, Dubey et al. 2006. 17. Crocidura suaveolens 8, Spain, Candelario, AY843492, Dubey et al. 2006. 18. Crocidura suaveolens 9, Iran, Gilan, DQ059023, Dubey et al. 2006. 19. Crocidura suaveolens 10, Azebaijan, AY843487, Dubey et al. 2006. 20. Crocidura russula, Switzerland, La Côte, AY769264, Fontanillas et al. 2005. 21. Crocidura shantungensis 1, South Korea, Cheju Is., AB077077, Ohdachi et al. 2004. 22. Crocidura shantungensis 2, South Korea, Kyungju, AB077079, Ohdachi et al. 2004. 23. Crocidura suaveolens 1 , Austria, Wien, AB077280, Ohdachi et al. 2004. 24. Crocidura suaveolens 11, China, Mosuowan, AB077087, Ohdachi et al. 2004. 25. Crocidura suaveolens 12, Ukraine, Sevastopol, AY843475, Dubey et al. 2006. 26. Crocidura lasiura, Russia, Ussuriisk, AB077071, Ohdachi et al. 2004. 27. Crocidura dsinezumi, Japan, Fukuoka, AB077275, Ohdachi et al. 2004. 28. Crocidura attenuata 1, Vietnam, Mt. Tay Con Linh, AB175082, Ohdachi et al. 2006. 29. Crocidura wuchihensis, Vietnam, Mt. Tay Con Linh, AB175084, Ohdachi et al. 2006. 30. Crocidura horsfieldi, Thailand, Kanchana Buri, AB175082, Ohdachi et al. 2006. 31. Crocidura watasei, Japan, Tokunoshima Is., AB077074, Ohdachi et al. 2006. 32. Crocidura fuliginosa, Vietnam, Mt. Tay Con Linh, AB175079, Ohdachi et al. 2006. 33. Crocidura attenuata 2, Taiwan, Nantou Co., AB175081, Ohdachi et al. 2006. 34. Crocidura nigripes, Indonesia, Sulawesi, DQ059024, Ohdachi et al. 2006. 35. Crocidura brunnea, Indonesia, Java, DQ059025, Ohdachi et al. 2006. 36. Crocidura orii, Japan, Amami-ohshima Is., AB175087, Ohdachi et al. 2006. 37. Crocidura kurodai, Taiwan, Nantou Co., AB175086, Ohdachi et al. 2006. 